\title{
Fractional Mellin Transform for Standard Functions
}

\author{
Vijaya Patil $^{1}$, Narhari Patil ${ }^{2}$ \\ ${ }^{1}$ Amravati University, Assistant Professor, Department of Applied Sciences and Humanities, Padm.Dr.V.B.Kolte College of Engineering, \\ Malkapur, Dist.:Buldana 443101 \\ ${ }^{2}$ Amravati University, HOD, Department of Applied Sciences and Humanities (Mathematics), \\ Shri Sant Gajanan Maharaj College of Engineering, Shegaon.
}

\begin{abstract}
Mellin transform is one of the members of an Integral transform. It is widely used in computer science for the analysis of algorithms of its scale invariance property. In mathematics, it may be regarded as the multiplicative version of the two-sided Laplace transform. In this paper we discuss the fractional Mellin transform with some basic properties. Also we prove fractional Mellin transform for Some Standard Functions. To illustrate the advantages and use of this transformation, example of differential equation has been solved at the end.
\end{abstract}

Keywords: Mellin transform, Laplace transform, Fractional Mellin Transform, Euleres Cauchy Differential equation

\section{Introduction}

Laplace transform is widely used by Engineers and mathematicians as a method of solving linear differential and integral equation [1]. Laplace operator is used to find the fractional Mellin transform The Mellin contribution gives a large place to the theory of analytic functions and relies essentially on Cauchy's theorem. More recently, traditional applications have been enlarged and new ones have emerged [2]-[6].In the present paper we discuss the fractional Mellin transform with some basic properties. And we illustrate fractional Mellin transform for Some Standard Functions. In the application part we solve the differential equations by using fractional Mellin transform.

\section{Fractional Mellin Transform}

(Preliminary results)

Mellin transform is closely related to an extended form of Laplace transform.

Let $f(x)$ be given function of ' $x$ ' which is defined for all $x \geq 0$ and , se is a parameter

$L[f(x), s, 0, \infty]=\int_{0}^{\infty} e^{-s x} f(x) d x$

The change of variable defined by

$x=-\log a t, d x=-\frac{d t}{t}, a>0$

If $x=0$ then $t=1 / a$ and if $x=\infty$ then $\mathrm{t}=0$, transforms the integral (1) into:

$L\left[f(-\log a t), s, 0, \frac{1}{a}\right]=\int_{0}^{1 / a} e^{-s(-\log a t)} f(-\log a t)\left(-\frac{d t}{t}\right)$

$L\left[f(t), s, 0, \frac{1}{a}\right]=\int_{0}^{1 / a} a^{s} t^{s-1} f(t) d t$

one recognizes in (4) the Fractional Mellin transform of $f(t)$ in the range $\left[0, \frac{1}{\mathrm{a}}\right]$, $\therefore M\left[f(t), s, 0, \frac{1}{a}\right]=\int_{0}^{1 / a} a^{s} t^{s-1} f(t) d t, a>0$

This can be written symbolically as:

$$
L[f(x), s, 0, \infty]=M\left[f(t), s, 0, \frac{1}{a}\right]
$$

\section{Properties}

P1] Linearity Property:

Let $\alpha, \beta, \gamma$ be any arbitrary constants and $f, g, h$ are functions of , $f^{\text {ce }}$.

$$
\begin{aligned}
& \text { If } M\left[f(t), s, 0, \frac{1}{a}\right]=\int_{0}^{1 / a} a^{s} t^{s-1} f(t) d t \text {, then } \\
& \quad M\left[\alpha f(t)+\beta g(t)-\gamma h(t), s, 0, \frac{1}{a}\right]=\alpha M\left[f(t), s, 0, \frac{1}{a}\right]+ \\
& \beta M\left[g(t), s, 0, \frac{1}{a}\right]-\gamma M\left[h(t), s, 0, \frac{1}{a}\right]
\end{aligned}
$$

P2] Scaling Property:

Let,$\beta^{\text {ee }}$ be any scalar and $f$ is any functions of , $f^{\prime}$.

$$
M\left[f(\beta t), s, 0, \frac{1}{a}\right]=\beta^{-s} M\left[f(P), s, 0, \frac{\beta}{a}\right],
$$

using the substitution $\beta t=P, \beta>0$.

P3] Shifting property:

$M\left[t^{n} f(t), s, 0, \frac{1}{a}\right]=M\left[f(t), s+n, 0, \frac{1}{a}\right]$

P4] Second shifting property:

$$
\begin{aligned}
& \text { If } M\left[f(t), s, 0, \frac{1}{a}\right]=\int_{0}^{1 / a} a^{s} t^{s-1} f(t) d t, \text { then } \\
& M\left[f(t-b) U(t-b), s, 0, \frac{1}{a}\right]=M\left[f(p), s-b, \frac{1}{a}-b\right]
\end{aligned}
$$

Proof:

$M\left[f(t-b) U(t-b), s, 0, \frac{1}{a}\right]=\int_{0}^{1 / a} a^{s} t^{s-1} f(t-b) U(t-b) d t$

Substituting $p=t-b$ we get, 


\section{International Journal of Science and Research (IJSR) \\ ISSN (Online): 2319-7064}

Index Copernicus Value (2015): 78.96 | Impact Factor (2015): 6.391

$M\left[f(t-b) U(t-b), s, 0, \frac{1}{a}\right]$

$=\int_{-b}^{\frac{1}{a}-b} a^{s}(p+b)^{s-1} f(p) U(p) d p$

$=\int_{-b}^{\frac{1}{a}-b} a^{s}(p+b)^{s-1} f(p) d p$, as $U(t-b)=1$ when $t>b$

$=M\left[f(p), s-b, \frac{1}{a}-b\right] \quad$ with Kernel $(p+b)^{s-1}$

P5] Derivative property:

The Mellin type Integral transform of $\mathrm{n}^{\text {th }}$ order derivative of $f(t)$ with respect to $t$ :

1] First order derivative:

If $M\left[f(t), s, 0, \frac{1}{a}\right]=\int_{0}^{1 / a} a^{s} t^{s-1} f(t) d t$, then

$M\left[f^{\prime}(t), s, 0, \frac{1}{a}\right]=(1-s) M\left[f(t), s-1,0, \frac{1}{a}\right]+a f\left(\frac{1}{a}\right)$

2] Second order derivative:

$M\left[f^{\prime \prime}(t), s, 0, \frac{1}{a}\right]=(1-s)(2-s) M\left[f(t), s-2,0, \frac{1}{a}\right]+(1-$
$s) a^{2} f\left(\frac{1}{a}\right)+a f^{\prime}\left(\frac{1}{a}\right)$

In general,

$M\left[f^{n}(t), s, 0, \frac{1}{a}\right]=$

$(1-s)(2-s) \ldots(n-s)$

$M[f(t), s-n, 0,1 a$

]$+(1-s) 2-s \ldots(n-1-s)$ anf $1 a+a$ fn $-11 a$

This is the fractional Mellin Integral transform of $n^{\text {th }}$ order derivative of $f(t)$.

\section{Application on Some Standard Functions:}

The Fractional Mellin transform is given by

$$
\begin{aligned}
M\left[f(t), s, 0, \frac{1}{a}\right] & =\int_{0}^{1 / a} a^{s} t^{s-1} f(t) d t \\
1] M\left[t^{n}, s, 0, \frac{1}{a}\right] & =\int_{0}^{1 / a} a^{s} t^{s-1} t^{n} d t \\
& =\int_{0}^{1 / a} a^{s} t^{(s+n)-1} d t \\
& =a^{s}\left[\frac{t^{s+n}}{s+n}\right]_{0}^{1 / a} \\
M\left[t^{n}, s, 0, \frac{1}{a}\right] & =\frac{\left(\frac{1}{a}\right)^{n}}{s+n}
\end{aligned}
$$

2] For Exponential Expansion:

$$
\begin{gathered}
M\left[e^{t}, s, 0, \frac{1}{a}\right]=\int_{0}^{1 / a} a^{s} t^{s-1} e^{t} d t \\
=a^{s} \int_{0}^{1 / a} t^{s-1}\left[1+\frac{t}{1 !}+\frac{t^{2}}{2 !}+\frac{t^{3}}{3 !}+\cdots\right] d t \\
=a^{s} \int_{0}^{1 / a}\left[t^{s-1}+\frac{t^{s}}{1 !}+\frac{t^{s+1}}{2 !}+\frac{t^{s+2}}{3 !}+\cdots\right] d t \\
=a^{s}\left[\frac{t^{s}}{s}+\frac{t^{s+1}}{1 !(s+1)}+\frac{t^{s+2}}{2 !(s+2)}+\frac{t^{s+3}}{3 !(s+3)} \cdots\right]_{0}^{1 / a} \\
=\frac{1}{s}+\frac{1}{1 !(s+1)}\left(\frac{1}{a}\right)+\frac{1}{2 !(s+2)}\left(\frac{1}{a}\right)^{2}+\frac{1}{3 !(s+3)}\left(\frac{1}{a}\right)^{3}+\cdots
\end{gathered}
$$

3] For Sine Expansion:

$$
\begin{aligned}
M\left[\sin a t, s, 0, \frac{1}{a}\right] & =\int_{0}^{1 / a} a^{s} t^{s-1} \sin a t d t \\
& =a^{s} \int_{0}^{1 / a} t^{s-1}\left[a t-\frac{(a t)^{3}}{3 !}+\frac{(a t)^{5}}{5 !}-\cdots\right] d t \\
& =a^{s} \int_{0}^{1 / a}\left[a t^{s}-\frac{a^{3}}{3 !} t^{s+2}+\frac{a^{5}}{5 !} t^{s+4}-\cdots\right] d t \\
& =a^{s}\left[\frac{a}{s+1} t^{s+1}-\frac{a^{3}}{3 !} \frac{1}{s+3} t^{s+3}+\right.
\end{aligned}
$$

$a 55 ! 1 s+5 t s+5 \ldots 01 a$

$$
\begin{aligned}
& =a^{s}\left[\frac{a}{s+1} \frac{1}{a^{s+1}}-\frac{a^{3}}{3 !} \frac{1}{s+3} \frac{1}{a^{s+3}}+\frac{a^{5}}{5 !} \frac{1}{s+5} \frac{1}{a^{s+5}} \ldots\right] \\
& =\frac{1}{s+1}-\frac{1}{3 !(s+3)}+\frac{1}{5 !(s+5)}-\cdots
\end{aligned}
$$

4] For Cosine Expansion:

$$
\begin{aligned}
M\left[\cos a t, s, 0, \frac{1}{a}\right] & =\int_{0}^{1 / a} a^{s} t^{s-1} \cos a t d t \\
& =a^{s} \int_{0}^{1 / a} t^{s-1}\left[1-\frac{(a t)^{2}}{2 !}+\frac{(a t)^{4}}{4 !}-\cdots\right] d t \\
& =a^{s} \int_{0}^{1 / a}\left[t^{s-1}-\frac{a^{2}}{2 !} t^{s+1}+\frac{a^{4}}{4 !} t^{s+3}-\cdots\right] d t \\
& =a^{s}\left[\frac{t^{s}}{s}-\frac{a^{2}}{2 !} \frac{t^{s+2}}{s+2}+\frac{a^{4}}{4 !} \frac{t^{s+4}}{s+4}-\cdots\right]_{0}^{1 / a} \\
& =\frac{1}{s}-\frac{1}{2 !(s+2)}+\frac{1}{4 !(s+4)}-\cdots
\end{aligned}
$$

5] For inverse Tangent Expansion:

$$
\begin{aligned}
M\left[\tan x^{-1} a t, s,\right. & \left.0, \frac{1}{a}\right]=\int_{0}^{1 / a} a^{s} t^{s-1} \tan ^{-1} a t d t \\
& =a^{s} \int_{0}^{1 / a} t^{s-1}\left[a t-\frac{(a t)^{3}}{3}+\frac{(a t)^{5}}{5}-\cdots\right] d t \\
& =a^{s} \int_{0}^{1 / a}\left[a t^{s}-\frac{a^{3}}{3} t^{s+2}+\frac{a^{5}}{5} t^{s+4}-\cdots\right] d t \\
& =a^{s}\left[\frac{a}{s+1} t^{s+1}-\frac{a^{3}}{3} \frac{1}{s+3} t^{s+3}+\right.
\end{aligned}
$$

$a 551 s+5 t s+5 \ldots .01 a$

$$
\begin{aligned}
& =a^{s}\left[\frac{a}{s+1} \frac{1}{a^{s+1}}-\frac{a^{3}}{3} \frac{1}{s+3} \frac{1}{a^{s+3}}+\frac{a^{5}}{5} \frac{1}{s+5} \frac{1}{a^{s+5}} \ldots\right] \\
& =\frac{1}{s+1}-\frac{1}{3(s+3)}+\frac{1}{5(s+5)}-\cdots
\end{aligned}
$$

6] For Logarithmic Expansion:

$$
\begin{gathered}
M\left[f(t), s, 0, \frac{1}{a}\right]=\int_{0}^{1 / a} a^{s} t^{s-1} f(t) d t \\
M\left[\log (1+t), s, 0, \frac{1}{a}\right]=\int_{0}^{1 / a} a^{s} t^{s-1} \log (1+t) d t \\
=a^{s} \int_{0}^{1 / a} t^{s-1}\left[t-\frac{t^{2}}{2}+\frac{t^{3}}{3}-\cdots\right] d t \\
=a^{s} \int_{0}^{1 / a}\left[t^{s}-\frac{t^{s+1}}{2}+\frac{t^{s+2}}{3}-\cdots\right] d t \\
=a^{s}\left[\frac{t^{s+1}}{(s+1)}-\frac{t^{s+2}}{2(s+2)}+\frac{t^{s+3}}{3(s+3)}-\cdots\right]_{0}^{1 / a} \\
=\frac{1}{(s+1)}\left(\frac{1}{a}\right)
\end{gathered}
$$

\section{Application to Differential Equation}

Derivative multiplied by independent variable is given by, 


\section{International Journal of Science and Research (IJSR) \\ ISSN (Online): 2319-7064}

Index Copernicus Value (2015): 78.96 | Impact Factor (2015): 6.391

$$
\begin{aligned}
& M\left[t^{n} f^{n}(t), s, 0, \frac{1}{a}\right]=\int_{0}^{1 / a} a^{s} t^{s-1} t^{n} f^{n}(t) d t \\
& =\int_{0}^{1 / a} a^{s} t^{s+n-1} f^{n}(t) d t a^{s}\left\{\left[t^{s+n-1} f^{n-1}(t)\right]_{0}^{1 / a}-\right. \\
& s+n-101 a t s+n-2 f n-1 t d t \\
& =a^{s}\left\{\left(\frac{1}{a}\right)^{s+n-1} f^{n-1}\left(\frac{1}{a}\right)-(s+n-1)\left(\frac{1}{a}\right)^{s+n-2} f^{n-2}\left(\frac{1}{a}\right)+\right. \\
& \left.(s+n-1)(s+n-2) \int_{0}^{1 / a} t^{s+n-3} f^{n-2}(t) d t\right\} \\
& =\left(\frac{1}{a}\right)^{n-1} f^{n-1}\left(\frac{1}{a}\right)-(s+n-1)\left(\frac{1}{a}\right)^{n-2} f^{n-2}\left(\frac{1}{a}\right)+ \\
& (s+n-1)(s+n-2) \int_{0}^{1 / a} a^{s} t^{s+n-3} f^{n-2}(t) d t \\
& =\left(\frac{1}{a}\right)^{n-1} f^{n-1}\left(\frac{1}{a}\right)-(s+n-1)\left(\frac{1}{a}\right)^{n-2} f^{n-2}\left(\frac{1}{a}\right)+ \\
& \quad(s+n-1)(s+n-2) M\left[t^{n-2} f^{n-2}(t), s, 0, \frac{1}{a}\right]
\end{aligned}
$$

Appling this rule until the Mellin transform of $\mathrm{n}$ the derivative of $f(t)$ is given by

$$
\begin{gathered}
M\left[t^{n} f^{n}(t), s, 0, \frac{1}{a}\right]=\left(\frac{1}{a}\right)^{n-1} f^{n-1}\left(\frac{1}{a}\right)-(s+n- \\
11 a n-2 f n-21 a+\frac{\sqrt{s+n}}{\sqrt{S}} M[f t, s, 0,1 a] \\
\text { Where } \frac{\sqrt{s+n}}{\Gamma_{s}}=(s+n-1)(s+n-2) \ldots \ldots . .(s+1) s
\end{gathered}
$$

Ex.1] $t^{2} \frac{d^{2} x}{d t^{2}}+t \frac{d x}{d t}=\log t$

$$
\begin{aligned}
M\left[t^{2} x^{\prime \prime}(t), s, 0, \frac{1}{a}\right] & =\int_{0}^{1 / a} a^{s} t^{s-1} t^{2} x^{\prime \prime}(t) d t \\
& =\int_{0}^{1 / a} a^{s} t^{s+1} x^{\prime \prime}(t) d t \\
= & a^{s}\left\{\left[t^{s+1} x^{\prime}(t)\right]_{0}^{1 / a}-(s+1) \int_{0}^{1 / a} t^{s+2} x^{\prime}(t) d t\right\} \\
= & \frac{1}{a} x^{\prime\left(\frac{1}{a}\right)}-(s+1) x\left(\frac{1}{a}\right)+s(s+1) M\left[x(t), s, 0, \frac{1}{a}\right]
\end{aligned}
$$

Also,

$$
\begin{aligned}
M\left[t x^{\prime}(t), s, 0, \frac{1}{a}\right] & =\int_{0}^{1 / a} a^{s} t^{s-1} t x^{\prime}(t) d t \\
& =\int_{0}^{1 / a} a^{s} t^{s} x^{\prime}(t) d t \\
& =a^{s}\left\{\left[t^{s} x(t)\right]_{0}^{1 / a}-(s) \int_{0}^{1 / a} t^{s-1} x(t) d t\right\} \\
= & x\left(\frac{1}{a}\right)-s M\left[x(t), s, 0, \frac{1}{a}\right] \\
M\left[(\log t), s, 0, \frac{1}{a}\right] & =\int_{0}^{1 / a} a^{s} t^{s-1}(\log t) d t \\
= & a^{s}\left\{\left[(\log t) \frac{t^{s}}{s}\right]_{0}^{1 / a}-\frac{1}{t} \int_{0}^{1 / a} \frac{t^{s}}{s} d t\right\} \\
= & a^{s} \frac{1}{s} \log \frac{1}{a}\left(\frac{1}{a}\right)^{s}-\frac{1}{s}\left[\frac{t^{s+1}}{t(s+1)}\right]_{0}^{1 / a}
\end{aligned}
$$

$$
=\frac{1}{s} \log \frac{1}{a}-\frac{1}{s(s+1)}
$$

Therefore the fractional Mellin transform of the given equation is,

$$
M\left[x(t), s, 0, \frac{1}{a}\right]=\frac{1}{s^{2}}\left[\frac{1}{s} \log \frac{1}{a}-\frac{1}{s(s+1)}-\frac{1}{a} x^{\prime}\left(\frac{1}{a}\right)+s x\left(\frac{1}{a}\right)\right]
$$

Ex.2] The Cauchy"s differential equation is,

$$
t^{2} \frac{d^{2} x}{d t^{2}}+t \frac{d x}{d t}+x(t)=0
$$

Taking fractional Mellin transform, we get

$$
\begin{gathered}
\frac{1}{a} x^{\prime}\left(\frac{1}{a}\right)-(s+1) x\left(\frac{1}{a}\right)+s(s+1) M\left[x(t), s, 0, \frac{1}{a}\right]+x\left(\frac{1}{a}\right)- \\
s M\left[x(t), s, 0, \frac{1}{a}\right]+M\left[x(t), s, 0, \frac{1}{a}\right]=0 \\
\therefore M\left[x(t), s, 0, \frac{1}{a}\right]=\frac{1}{s^{2}+1}\left[s x\left(\frac{1}{a}\right)-\frac{1}{a} x^{\prime}\left(\frac{1}{a}\right)\right]
\end{gathered}
$$

\section{Conclusion}

In this paper some standard functions are solved by using fractional Mellin Transform. We have obtained interesting results for Expansion formulae. To illustrate the advantages and use of the transforms some differential equation has been solved.

\section{References}

[1] J. Bertrand, P. Bertrand and J. P. Ovarlez , "The Transforms and Applications Handbook" Florida, USA: CRC Press Inc., 1995,pp. 1-60.

[2] N.A.Patil and V. N. Patil , "Roll Of Double Singly Infinite Laplace Transform (SIL-FM Relation) In Engineering" International Journal of Applied Mathematics \& Statistical Sciences (IJAMSS) Vol. 3, Issue 4, Jul 2014, pp. 2532@ IASET

[3] S.M. Khairnar, R.M.Pise and J.N.Salunke , "Fractional Mellin Integral Transform in (0, 1/a)" International Journal of Scientific and Research Publications, Vol.2, ISSN 22503153, I(5), May 2012.

[4] V. N. Mahalle, A. S. Gudadhe and R. D. Taywade , "Generalization of Linear Scale Invariant System inthe Fractional Domain and Some Properties of Fractional Complex Mellin Transform”, Vol. 6, 2011.

[5] V. N. Patil and N. A. Patil , "Usage of Mellin transform in analogous system and network analysis" IORD Journal of Science \& Technology Volume 2, Issue 3 pp. 01-04 (MARAPR 2015)

[6] Y. Peng, Y. Wang, X. Zuo and L. Gong, "Properties of Fractional Mellin Transform"Advances in Information Sciences and Service Sciences(AISS)Vol.5, Number5, pp.90-96, March 2013. 